\title{
Cardiotoxicity evaluation in pediatric patients with acute lymphoblastic leukemia - results of prospective study
}

\author{
Letitia-Elena Radu1,2, Ioana Ghiorghiu ${ }^{1,3}$, Alina Oprescu ${ }^{4}$, Dan Dorobantu ${ }^{5}$, Constantin Arion ${ }^{1,2}$, \\ Anca Colita ${ }^{1,2}$
}

${ }^{1}$ "Carol Davila" University of Medicine and Pharmacy, ${ }^{2}$ Fundeni Clinical Institute, Department of Pediatric Hema-

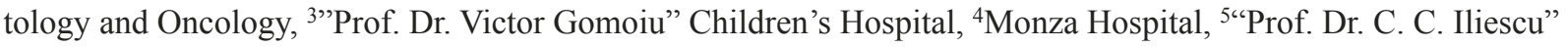
Cardiovascular Diseases Institute, Bucharest, Romania

\begin{abstract}
Aim: The chemotherapy protocol for acute lymphoblastic leukemia (ALL) uses low doses of anthracyclines (AC), generally associated with subclinical cardiotoxicity. The aim of our study was to evaluate the serum biomarkers and echocardiography parameters in children with ALL treated with AC in order to determine the most useful element for early detection of cardiotoxicity. Material and methods: In this prospective study, troponin I (TnI) and heart-type fatty acid binding protein (HFABP) were assessed five times during the first year after the onset of ALL. Serial Tissue Doppler Imaging and conventional cardiac echography were performed by two pediatric cardiologists (intraclass correlation coefficient over 0.85 for all measurements) in three periods during the study protocol. Results: We evaluated 48 children with ALL. TnI increased during therapy, without returning to baseline values one year after diagnosis. HFABP did not show significant changes during the study protocol. Left ventricle outflow tract time-velocity integral and peak systolic septal mitral annulus velocity decreased during chemotherapy and returned to baseline levels at one year after diagnosis, while peak systolic tricuspid annulus velocity and excursion, maintained a descending tendency. Early filling transmitral flow velocity and E/A ratio were also transiently influenced by chemotherapy. Conclusions: The study showed signs of transient cardiotoxicity in the left ventricle and diastolic parameters after chemotherapy, compared to right ventricle parameters which maintained low values even one year after diagnosis. TnI proved to be directly proportional to chemotherapy doses but HFABP was not useful in this setting.
\end{abstract}

Keywords: acute lymphoblastic leukemia; cardiotoxicity; biomarkers; Tissue Doppler Imaging

\section{Introduction}

Acute leukemia represents $25-30 \%$ of all pediatric neoplasms and acute lymphoblastic leukemia (ALL) comprises $80 \%$ of cases [1]. At present, the overall sur-

Received 07.06.2019 Accepted 18.10.2019

Med Ultrason

2019, Vol. 21, No 4, 449-455

Corresponding author: Letitia-Elena Radu

258 Fundeni Road, building A, floor 6 , Pediatrics Department, Bucharest, Romania

E-mail: letitia_radu@yahoo.com

Phone: +40 721115203 vival rates of ALL patients are between 80-90\% [2], being the most successful story of modern multi-agent chemotherapy [3].

The harmful effect of chemotherapy on the heart muscle is well known, the most impugned being the anthracyclines (AC) class [4], which triggers the prototype for type I chemotherapy-induced cardiotoxicity (CIC), characterized by dose-dependent irreversible myocardial cell death [5]. Three types of CIC were described: acute (one week after AC administration, in less than $1 \%$ of patients), early-onset (during the first year after diagnosis, in approximately $2 \%$ of patients) and late-onset (one year after diagnosis) [6]. 
The gold standard for CIC diagnosis is serial ultrasound [7], transthoracic echocardiography being the recommended method [8]. Due to frequent asymptomatic cardiotoxicity, more sensitive methods of diagnosis are required [9] and Tissue Doppler Imaging (TDI) provides a promising technique for evaluating cancer patients [10]. Cardiac biomarkers may represent an operator-independent alternative for CIC detection, but an optimal schedule of biomarker assessments remains unclear [11]. Alongside cardiac troponins and natriuretic peptides, novel biomarkers emerge, such as high-sensitivity Creactive protein and fatty acid binding protein [12]. Clear recommendations remain to be established.

The aim of our study was to assess the early-onset CIC after low-dose doxorubicin-based treatment in children diagnosed with ALL, in a single pediatric hematologyoncology center, by monitoring the changes of troponin I (TnI) and heart-type binding protein (HFABP) levels and the parameters of conventional and TDI echocardiography over a period of one year after diagnosis.

\section{Material and methods}

\section{Patients}

A prospective non-randomized observational study was conducted between February 2015 and November 2017 in the Department of Pediatric Hematology and Oncology. All legal guardians signed an informed consent form. The study and the informed consent form were preapproved by the local Ethics Committee. Patients 1 to 18 years of age, newly diagnosed with ALL and treated according to BFM ALL IC 2009 protocol [13], plus minimal residual disease monitoring on days 15,33 and 78, were enrolled. Based on risk stratification imposed by BFM ALL IC 2009 protocol [13] the patients received $8-10$ doses of $30 \mathrm{mg} / \mathrm{m}^{2} \mathrm{AC}$, adapted to the neutrophil count. The first dose of $\mathrm{AC}$ was given on day 8 and the last one at 9 months after diagnosis. The discontinuation criteria from the study were death of any cause, recurrence of the disease, emigration (at any moment during the monitoring period).

\section{Cardiac biomarkers}

Second generation TnI was assayed on Tosoh Bioscience, AIA-1800 (Immunoassay), normal range 0-0.05 $\mathrm{pg} / \mathrm{dl}$, sensitivity $0.01 \mathrm{pg} / \mathrm{dl}$. HFABP was analyzed on calibrated ELISA plate reader using the human HFABP kit (Hycult biotech), normal range 0-1.6 ng/ml, sensitivity $0.102 \mathrm{ng} / \mathrm{ml}$. Five blood samples were obtained: at diagnosis (TnI1, HFABP1), one hour after the first AC dose (TnI2, HFABP2), before the last AC dose (TnI3, HFABP3), one hour after the last AC dose (TnI4, HFABP4) and one year after diagnosis (TnI5, HFABP5).

\section{Echocardiographic evaluation}

Echocardiographic evaluation, including TDI, was performed at 3 different times: at diagnosis, after all AC doses and one year after diagnosis. The scans were performed and analyzed online on a Vivid E95 (GE Vingmed Imaging, Horten, Norway) by two pediatric cardiologists with more than 10 years of experience in pediatric echocardiography, blinded to patients' status. In order to determine intra/inter variability, 10 echocardiographic examinations were selected and reevaluated. An intraclass correlation factor over 0.85 for all measurements was obtained. The following measurements were performed: left ventricle ejection fraction (LVEF) with the M mode Teichholz method, LV outflow tract time-velocity integral (TVI), tricuspid annular plane systolic excursion (TAPSE), peak systolic septal mitral annulus velocity (SS), peak systolic lateral mitral annulus velocity (SL) and right ventricle (RV)-peak systolic tricuspid annulus velocity (RVS), early filling transmitral flow velocity (E), late filling transmitral flow velocity (A), E-wave deceleration time (EDT), isovolumic relaxation time (IVRT), E/A. All acquisitions and measurements were performed in concordance with current recommendations [14].

\section{Statistical analysis}

The statistical analysis was performed using IMB Statistical Package for Social Sciences, SPSS 25. For bivariate analysis Mann-Whitney U and Kruskall Wallis tests were used and the results were reported as median and interquartile range (IQR). A $p$ value of $<0.05$ was considered statistically significant, unless otherwise stated. For the evaluation of biomarkers and ultrasound parameters Friedman's test was used, with post-hoc analysis by Wilcoxon Signed Rank, applying Bonferroni correction. The representation was done using parallel graphics.

\section{Results}

During the enrolment period, 70 children were diagnosed with ALL. After applying the discontinuation criteria, 48 patients finalized the study protocol. The demographic and hematologic data of these patients are detailed in Table I.

The serum levels of the two cardiac biomarkers are presented in Table II. After applying Boferroni correction, $p<0.005$ was considered significant for serum biomarkers analysis. No significant changes in HFABP values were found during the study protocol $(\mathrm{p}=0.37)$. As shown in figure $1, \mathrm{TnI}$ increased after therapy compared to baseline $(\mathrm{p}<0.001)$. TnI5 levels decreased compared to TnI4 (no statistical significance, $\mathrm{p}=0.118$ ), but remained higher than baseline values. There were no correlations 
Table I. Demographic and hematologic data of patients

\begin{tabular}{|c|c|c|}
\hline & & $\begin{array}{l}\text { Number of } \\
\text { patients }(\%)\end{array}$ \\
\hline Gender male: female & & $\begin{array}{l}29: 19 \\
(60.4: 39.6)\end{array}$ \\
\hline \multirow[t]{3}{*}{ Age groups } & $1-5$ years & $24(50)$ \\
\hline & $6-10$ years & $11(22.9)$ \\
\hline & $>10$ years & $13(27.1)$ \\
\hline \multirow{4}{*}{$\begin{array}{l}\text { Leucocyte count } \\
\text { at diagnosis }\end{array}$} & $<10 \times 10^{9} / \mathrm{L}$ & $22(45.8)$ \\
\hline & $10-20 \times 10^{9} / \mathrm{L}$ & $11(22.9)$ \\
\hline & $20-50 \times 10^{9} / \mathrm{L}$ & $5(10.4)$ \\
\hline & $>50 \times 10^{9} / \mathrm{L}$ & $10(20.9)$ \\
\hline \multirow[t]{2}{*}{ Morphology } & $\mathrm{L} 1$ & $44(91.7)$ \\
\hline & L2 & $4(8.3)$ \\
\hline \multirow[t]{2}{*}{ Immunophenotype } & B-cell & $39(81.3)$ \\
\hline & T-cell & $9(18.7)$ \\
\hline \multirow[t]{3}{*}{ Cytogenetics } & abnormalities & $12(25)$ \\
\hline & no metaphases & $20(41.7)$ \\
\hline & normal karyotype & $16(33.3)$ \\
\hline \multirow[t]{4}{*}{ Molecular biology* } & negative & $32(66.7)$ \\
\hline & TEL-AML1 & $10(20.8)$ \\
\hline & E2A-PBX1 & $4(8.3)$ \\
\hline & BCR-ABL1 & $2(4.2)$ \\
\hline \multirow[t]{2}{*}{ Prednisone response ${ }^{* *}$} & good & $43(90.6)$ \\
\hline & poor & $5(9.4)$ \\
\hline \multirow[t]{3}{*}{ Risk groups } & standard risk & $19(39.6)$ \\
\hline & intermediate risk & $21(43.8)$ \\
\hline & high risk & $8(16.6)$ \\
\hline \multirow[t]{3}{*}{ Cumulative dose of $\mathrm{AC}$} & $<200 \mathrm{mg} / \mathrm{m}^{2}$ & $7(14.6)$ \\
\hline & $200-240 \mathrm{mg} / \mathrm{m}^{2}$ & $25(52.1)$ \\
\hline & $\geq 240 \mathrm{mg} / \mathrm{m}^{2}$ & $16(33.3)$ \\
\hline
\end{tabular}

* fusion genes analyzed: TEL-AML1, SIL-TAL1, E2A-PBX1, MLL-AF4, BCR-ABL1

** prednisone good response: $<1$ blast $\mathrm{x} 10^{9} / \mathrm{L}$ on day 8 ; prednisone poor response: $>1$ blast $\times 10^{9} / \mathrm{L}$ on day 8

Table II. The serum levels of troponin I (TnI) and heart-type binding protein (HFABP)

\begin{tabular}{lll}
\hline & TnI (pg/dl) & HFABP $(\mathbf{n g} / \mathbf{m l})$ \\
\hline $\begin{array}{l}\text { T1, before } \\
\text { the first AC dose }\end{array}$ & $0.01(0.01,0.01)$ & $1.9(1.71,2.18)$ \\
$\begin{array}{l}\text { T2, one hour after } \\
\text { the first dose }\end{array}$ & $0.01(0.01,0.01)$ & $1.91(1.75,2.27)$ \\
$\begin{array}{l}\text { T3, before } \\
\text { the last AC dose }\end{array}$ & $0.015(0.01,0.02)$ & $1.85(1.62,1.98)$ \\
$\begin{array}{l}\text { T4, one hour after } \\
\text { the last dose }\end{array}$ & $0.02(0.01,0.03)$ & $1.75(1.6,1.97)$ \\
$\begin{array}{l}\text { T5, one year after } \\
\text { diagnosis }\end{array}$ & $0.01(0.01,0.02)$ & $1.69(1.56,1.75)$ \\
\hline
\end{tabular}

The results are expressed as median value (interquartile range).

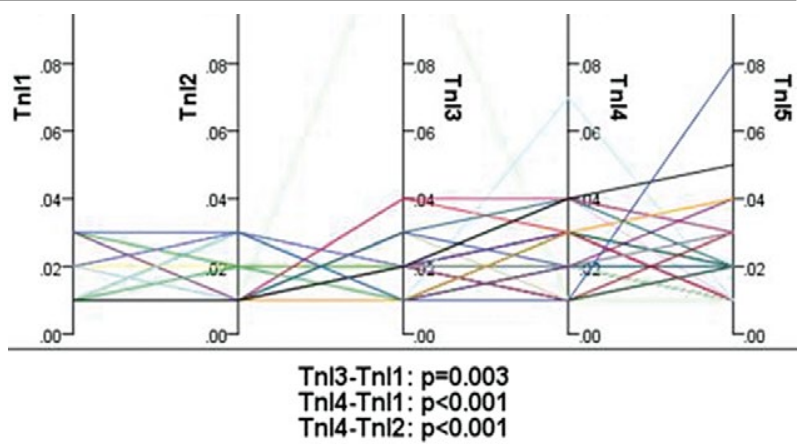

Fig 1. Parallel coordinates plot, showing the variability of TnI among all 48 patients at five timepoints; $\mathrm{TnI} 1=$ before the first $\mathrm{AC}$ dose; TnI 2 = one hour after the first $\mathrm{AC}$ dose; $\mathrm{TnI} 3=$ before the last $\mathrm{AC}$ dose; $\mathrm{TnI} 4=$ one hour after the last $\mathrm{AC}$ dose; TnI5 $=$ one year after diagnosis

between the two biomarkers at the end of treatment and cumulative dose of $\mathrm{AC}(\mathrm{p}=0.715$, respectively $\mathrm{p}=0.876)$.

Serial echocardiographic evaluations were performed on 46 patients; in two children, due to technical difficulties, the results were not considered for analysis (Table III).

Applying Boferroni correction, $\mathrm{p}<0.017$ was considered statistically relevant for echocardiographic evaluations. TVI and E decreased during therapy $(\mathrm{p}<0.001)$ and returned to pre-treatment values at one year after diagnosis $(p<0.001)$. SS decreased at the end of therapy $(\mathrm{p}=0.008)$ and slightly increased one year after diagnosis, with no statistical significance. E/A decreased after all AC doses $(p=0.014)$ and increased one year after diagnosis $(\mathrm{p}=0.026$, marginal $)$.

TAPSE decreased after treatment $(p<0.001)$ and maintained a lower value than baseline one year after diagnosis $(\mathrm{p}=0.002)$; RVS also decreased $(\mathrm{p}=0.006)$ and remained so one year after diagnosis $(\mathrm{p}=0.001)$.

No significant correlations between hematological tests, including morphological, cytogenetic or molecular subtypes, the two biomarkers and echocardiographic findings could be established (all $\mathrm{p}>0.05$ ).

\section{Discussion}

Troponin is considered to be the best suited cardiac biomarker for detecting CIC, cellular damage and apoptosis $[15,16]$, but also for subclinical cardiac dysfunction [17]. TnI is more feasible than the other cardiac troponins for early CIC diagnosis due to its kinetic curve [18], but the exact dynamic of troponin release into bloodstream is not well known $[5,12,19]$. Although several papers have been published regarding CIC evaluation by troponin measurements, there are only a few studies on this subject in pediatric ALL setting [20-29]. 
Table III. The echocardiographic parameters of the study group (46 patients)

\begin{tabular}{lllll}
\hline & At diagnosis & After all AC doses & 1 year after diagnosis & p \\
\hline LVEF, $\%$ & $63(60.5,65)$ & $64.5(60,67)$ & $62(60,65)$ & 0.833 \\
TVI, cm & $19.1(11.22,21.77)$ & $15.75(14.57,18.6)$ & $19.6(16.37,24.52)$ & $<\mathbf{0 . 0 0 1}$ \\
TAPSE, cm & $2.1(1.9,2.3)$ & $1.8(1.6,1.9)$ & $1.9(1.7,2.1)$ & $<0.001$ \\
$\mathrm{SS}, \mathrm{cm} / \mathrm{s}$ & $7.7(6.8,8.6)$ & $6.9(6,7.5)$ & $7(6,8)$ & 0.07 \\
$\mathrm{SL}, \mathrm{cm} / \mathrm{s}$ & $8.7(7.9,10.3)$ & $8.7(7,10)$ & $8(6.6,10)$ & 0.488 \\
$\mathrm{RVS}, \mathrm{cm} / \mathrm{s}$ & $14.4(12,15)$ & $12(10.8,13.5)$ & $12(10.4,13.1)$ & $\mathbf{0 . 0 0 2}$ \\
IVRT, ms & $50(41,63.5)$ & $44(38,61)$ & $57(43,69)$ & 0.173 \\
$\mathrm{E}, \mathrm{m} / \mathrm{s}$ & $0.9(0.8,1)$ & $0.8(0.7,0.9)$ & $0.9(0.9,1.1)$ & $\mathbf{0 . 0 0 1}$ \\
$\mathrm{A}, \mathrm{m} / \mathrm{s}$ & $0.6(0.5,0.7)$ & $0.6(0.5,0.7)$ & $0.7(0.5,0.8)$ & 0.936 \\
E/A & $1.5(1.2,1.9)$ & $1.4(1.2,1.6)$ & $1.4(1.2,1.9)$ & $\mathbf{0 . 0 3 1}$ \\
EDT, ms & $121(97.8,149.2)$ & $115(100.8,136)$ & $129(106,169)$ & 0.05 \\
\hline
\end{tabular}

The results are expressed as median value (interquartile range). A = late filling transmitral flow velocity; $\mathrm{E}=$ early filling transmitral flow velocity; EDT = E-wave deceleration time; risk; IVRT = isovolumic relaxation time; $\mathrm{LVEF}=$ left ventricle ejection fraction; $\mathrm{RVS}=$ peak systolic tricuspid annulus velocity; SL = peak systolic lateral mitral annulus velocity; SS = peak systolic septal mitral annulus velocity; TAPSE $=$ tricuspid annular plane systolic excursion; TVI = left ventricle outflow tract time-velocity integral

Different groups monitored large cohorts of children with ALL and concluded that TnI was predictable for myocardial injury [20-22]. There is evidence that TnI level is correlated with AC treatment in a dose-dependent manner [23-24] and the increase occurs 2-4 weeks after administration [25] but there are studies in which no changes in the troponin values during AC treatment were found [26-29]. In our study, in children with ALL receiving low $\mathrm{CD}$ of $\mathrm{AC}$, $\mathrm{TnI}$ increased in direct proportion with the number of doses $(p<0.001)$, only one hour after the administration. One year after ALL onset, TnI had the tendency to decrease, remaining higher compared with the baseline value, suggesting that subclinical lesions could be reversible in these patients.

There are no published data about HFABP levels in pediatric ALL patients and data provided by adult cohorts and mice studies are contradictory: two studies found increased levels after AC-based treatment [3031], while two showed no relevant changes [32-33]. We found no statistically significant variation in HFABP levels in children but, knowing that this biomarker decreases slowly as the cumulative dose of AC increases due to the HFABP gene suppression [34], a longer period of monitoring would be useful.

No significant changes in LVEF could be established in our study group, the parameter appearing to be insufficiently sensitive in detecting early signs of cardiac dysfunction after low-dose AC treatment, as previously mentioned [5,35-38].

Both in adults and children, TVI monitoring is recommended in several pathologies [39]. In a reference paper, normal values for TVI are proposed [40]. In our study TVI baseline values were within normal range and after
AC-based therapy the values decreased $(\mathrm{p}<0.001)$, suggesting that TVI is a sensitive and independent predictor for CIC, as Schmitt et al previous founded [41]. Our patients were evaluated also at one year after diagnosis, TVI returning to normal $(\mathrm{p}<0.001)$, showing a rapid, but transient global dysfunction.

The normal values for SS were established in measurements realized in an offline mode [42]. Our measurements (at baseline and one year after diagnosis) were performed in an online mode but we obtained similar results. We suggest that the decrease of the SS at the end of treatment $(\mathrm{p}=0.008)$, represents transient contractility dysfunction as the values obtained after 1 year after diagnosis return to normal, quite similar with previous published studies $[36,43,44]$.

Regarding SL, our results were similar to reported normal values $[10,42]$. Contraversely, some studies observed significant changes in SL after AC treatment [36]. The difference between SS and SL dynamics in our patients could suggest a higher sensitivity for contractile dysfunction at the interventricular septum level.

We evaluated the RV based on TAPSE, useful for global and longitudinal function, and RVS, which assesses longitudinal and contractile function. We found a decrease in both parameters at the end of therapy $(p<0.001, p=0.006)$, similar to other published data [4447]. However, another study did not describe any changes in TAPSE or RVS [48]. The decrease in RV parameters was maintained even one year after diagnosis, compared to LV parameters which returned to baseline levels. The distinct way of reacting to injury could be due to a different pattern of dysfunction regarding the two ventricles [44,49-50]. 
At present, diastolic dysfunction in children is not clarified; more to the point, the necessary parameters for its evaluation are not established [51]. Even though it is well known that hydric balance influences diastolic measurements, it is virtually impossible to adjust the evaluation protocol in ALL pediatric setting [52]. In our group, IVRT and A wave did not change during AC treatment, in opposition to other studies [53-55]. E and E/A ratio decreased during therapy $(\mathrm{p}<0.001, \mathrm{p}=0.014)$, suggesting a dysfunction in LV filling, similar results being encountered in different papers [54-55]. One year after diagnosis, $\mathrm{E}$ and $\mathrm{E} / \mathrm{A}$ ratio returned to baseline values. Conclusions regarding diastolic changes in this cohort cannot be drawn, probably due to the unreliable method of evaluation, considering the volemic status of the pediatric patients undergoing chemotherapy.

\section{Limitations}

The Teichholz method was used in order to obtain images in a shorter amount of time; the patients were immunocompromised and the majority under the age of 5 , therefore, not very cooperant. The Speckle tracking technique was not used for the same reasons. The echocardiographic protocol was focused on systolic ventricular function; only a few of the diastolic parameters were measured. The variation in parameters due to age was limited by mainly comparing the same patient at different time points.

\section{Conclusions}

The TnI levels decreased proportionally with anthracycline administration. Left ventricle and diastolic parameters (TVI, SS, E, E/A) decreased during therapy and returned to pre-treatment values one year after diagnosis. Right ventricle parameters (TAPSE, RVS) remained at lower values one year after diagnosis. HFABP, LVEF, SL, IVRT and A wave were not influenced during protocol. Further studies are required to establish a protocol for early diagnosis and monitoring of CIC in children with ALL in order to minimize acute and chronic cardiac side effects in cancer survivors.

\section{Conflict of interest: none}

\section{References}

1. Sági JC, Egyed B, Kelemen A, et al. Possible roles of genetic variations in chemotherapy related cardiotoxicity in pediatric acute lymphoblastic leukemia and osteosarcoma. BMC Cancer 2018;18:704.

2. Lee JW, Cho B. Prognostic factors and treatment of pediatric acute lymphoblastic leukemia. Korean J Pediatr 2017;60:129-137.
3. Radu LE, Beldiman A, Ghiorghiu I, Oprescu A, Arion C, Colita A. The use of biomarkers in detecting subclinical cardiotoxicity in doxorubicin-based treatment for paediatric patients with acute lymphoblastic leukaemia. Rev Romana Med Lab 2017;25:157-164.

4. Kim H, Kang HJ, Park KD, et al. Risk Factor Analysis for Secondary Malignancy in Dexrazoxane-Treated Pediatric Cancer Patients. Cancer Res Treat 2019;51:357-367.

5. Kim H, Chung WB, Cho KI, et al. Diagnosis, treatment, and prevention of cardiovascular toxicity related to anti-cancer treatment in clinical practice: an opinion paper from the working group on cardio-oncology of the Korean society of echocardiography. J Cardiovasc Ultrasound 2018;26:1-25.

6. Harake D, Franco VI, Henkel JM, Miller TL, Lipshultz SE. Cardiotoxicity in childhood cancer survivors: strategies for prevention and management. Future Cardiol 2012;8:647-670.

7. Zamorano JL, Lancellotti P, Rodriguez Munoz D, et al. 2016 ESC Position Paper on cancer treatments and cardiovascular toxicity developed under the auspices of the ESC Committee for Practice Guidelines: The Task Force for cancer treatments and cardiovascular toxicity of the European Society of Cardiology (ESC). Eur Heart J 2016;37:2768-2801.

8. Zito C, Longobardo L, Cadeddu C, et al. Cardiovascular imaging in the diagnosis and monitoring of cardiotoxicity: role of echocardiography. J Cardiovasc Med. 2016; 17(suppl 1):e35-e44.

9. Emren SV, Tuluce SY, Levent F, et al. Evaluation of Trastuzumab-induced early cardiac dysfunction using two-dimensional Strain Echocardiography. Med Ultrason 2015;17:496-502.

10. Yoldaş T, Yeşil Ş, Karademir S, et al. Evaluation of longterm cardiac side effects of anthracycline chemotherapy by conventional and non-conventional echocardiographic methods in childhood cancer survivors. Cardiol Young 2019;29:904-909.

11. Tian S, Hirshfield KM, Jabbour SK, et al. Serum biomarkers for the detection of cardiac toxicity after chemotherapy and radiation therapy in breast cancer patients. Front Oncol 2014;4:277.

12. Perez IE, Taveras Alam S, Hernandez GA, Sancassani R. Cancer Therapy-Related Cardiac Dysfunction: An Overview for the Clinician. Clin Med Insights Cardiol 2019;13:1179546819866445.

13. ALL IC-BFM 2009. Available at:http://www.bialaczka.org/ wp-content/uploads/2016/10/ALLIC_BFM_2009.pdf

14. Galderisi M, Plana JC, Edvardsen T, et al. Cardiac oncology. In: Lancellotti P, Cosyns B (eds). The EACVI Echo Handbook. Oxford Press. 2017;6:552-559.

15. Wallace KB, Hausner E, Herman E, et al. Serum troponins as biomarkers of drug-induced cardiac toxicity. Toxicol Pathol 2014;32:106-121.

16. Michel L, Rassaf T, Totzeck M. Biomarkers for the detection of apparent and subclinical cancer therapy-related cardiotoxicity. J Thorac Dis 2018;10(Suppl 35):S4282-S4295.

17. Shah KS, Yang EH, Maisel AS, Fonarow GC. The Role of Biomarkers in Detection of Cardio-toxicity. Curr Oncol Rep 2017;19:42. 
18. Christenson ES, James T, Agrawal V, Park BH. Use of biomarkers for the assessment of chemotherapy-induced cardiac toxicity. Clin Biochem 2015;48:223-235.

19. Gillespie HS, McGann CJ, Wilson BD. Noninvasive diagnosis of chemotherapy related cardiotoxicity. Curr Cardiol Rev 2011;7:234-244.

20. Lipshultz SE, Miller TL, Scully RE, et al. Changes in cardiac biomarkers during doxorubicin treatment of pediatric patients with high-risk acute lymphoblastic leukemia: associations with long-term echocardiographic outcomes. J Clin Oncol 2012;30:1042-1049.

21. El-Shitany NA, Tolba OA, El-Shanshory MR, EL-Hawary EE. Protective effect of carvedilol on adriamycin-induced left ventricular dysfunction in children with acute lymphoblastic leukemia. J Card Fail 2012;18:607-613.

22. Oztarhan K, Guler S, Aktas B, Arsian M, Salcioglu Z, Audogan $G$. The value of echocardiography versus cardiac troponin i levels in the early detection of anthracycline cardiotoxicity in childhood acute leukemia: Prospective evaluation of a 7-year-long clinical follow-up. Pediatr Hematol Oncol 2011;28:380-394.

23. Lee HS, Son CB, Shin SH, Kim YS. Clinical correlation between brain natriuretic peptide and anthracyclin-induced cardiac toxicity. Cancer Res Treat 2008;40:121-126.

24. Kunarajah K, Hennig S, Norris RLG, et al. Population pharmacokinetic modelling of doxorubicin and doxorubicinol in children with cancer: is there a relationship with cardiac troponin profiles? Cancer Chemother Pharmacol 2017;80:15-25

25. Krischke M, Hempel G, Völler S, et al. Pharmacokinetic and pharmacodynamic study of doxorubicin in children with cancer: results of a "European Pediatric Oncology Off-patents Medicines Consortium" trial. Cancer Chemother Pharmacol 2016;78:1175-1184.

26. Erkus B, Demirtas S, Yarpuzlu AA, Can M, Genc Y, Karaca L. Early prediction of anthracycline induced cardiotoxicity. Acta Paediatr 2007;96:506-509.

27. Xu XY, Huang MR, Tang JY, Zhang YQ, Wy YR, Zhou M. Evaluation of early monitoring of cardiotoxicity induced by anthracyclines. Zhongguo Dang Dai Er Ke Za Zhi 2011;13:490-494.

28. Soker M, Kervancioglu M. Plasma concentration on NTpro-BNP and cardiac troponin-I in relation to doxorubicininduced cardiomyopathy and cardiac function in childhood malignancy. Saudi Med J 2005;26:1197-1202.

29. Specchia G, Buquicchio C, Pansini N, et al. Monitoring of cardiac function on the basis of serum troponin I levels in patients with acute leukemia treated with anthracyclines. J Lab Clin Med 2005;145:212-220.

30. Ozturk G, Tavil B, Ozguner M, et al. Evaluation of Cardiac Markers in Children Undergoing Hematopoietic Stem Cell Transplantation. J Clin Lab Anal 2015;29:259262.

31. ElGhandour AH, Sorady ME, Azab S, ElRahman M. Human heart-type fatty acid-binding protein as an early diagnostic marker of doxorubicin cardiac toxicity. Hematol Rev 2009;1:e6.
32. Horacek JM, Vasatova M, Tichy M, Pudil R, Jebavy L, Maly J. The use of cardiac biomarkers in detection of cardiotoxicity associated with conventional and high-dose chemotherapy for acute leukemia. Exp Oncol 2010;32:9799.

33. Lai RC, Wang XD, Zhang X, Lin WQ, Rong TH. Heart fatty acid-binding protein may not be an early biomarker for anthracycline-induced cardiotoxicity in rabbits. Med Oncol 2012;29:2303-2308.

34. Sayed-Ahmed MM, Al-Shabanah OA, Hafez MM, Aleisa AM, Al-Rejaie SS. Inhibition of gene expression of heart fatty acid binding protein and organic cation-carnitine transporter in doxorubicin cardiomyopathic rat model. Eur J Pharmacol 2010;640:143-149.

35. Watts RG, George M, Johnson WH Jr. Pretreatment and routine echocardiogram monitoring during chemotherapy for anthracycline-induced cardiotoxicity rarely identifies significant cardiac dysfunction or alters treatment decisions: A 5-year review at a single pediatric oncology center. Cancer 2012;118:1919-1924.

36. Agha H, Shalaby L, Attia W, Abdelmohsen G, Aziz OA, Rahman MY. Early Ventricular Dysfunction After Anthracycline Chemotherapy in Children. Pediatr Cardiol 2016;37:537-544.

37. Chow EJ, Leger KJ, Bhatt NS, et al. Paediatric cardiooncology: epidemiology, screening, prevention, and treatment. Cardiovasc Res 2019;115:922-934.

38. Venneri L, Zoppellaro G, Khattar RS. Cardio-oncology: the role of advanced echocardiography in cancer patients. Expert Rev Cardiovasc Ther 2018;16:249-258.

39. Porter TR, Shillcutt SK, Adams MS, et al. Guidelines for the Use of Echocardiography as a Monitor for Therapeutic Intervention in Adults: A Report from the American Society of Echocardiography. J Am Soc Echocardiogr 2015;28:4056.

40. Solinski A, Klusmeier E, Horst JP, et al. Centile Curves for Velocity-Time Integral Times Heart Rate as a Function of Ventricular Length: The Use of Minute Distance Is Advantageous to Enhance Clinical Reliability in Children. J Am Soc Echocardiogr 2018;31:105-112.e2.

41. Schmitt K, Tulzer G, Merl M, et al. Early detection of doxorubicin and daunorubicin cardiotoxicity by echocardiography: Diastolic versus systolic parameters. Eur J Pediatr 1995;154:201-204.

42. Van der Hulst AE, Delgado V, ten Harkel AD, et al. Tissue Doppler imaging in the left ventricle and right ventricle in healthy children: normal age-related peak systolic velocities, timings, and time differences. Eur J Echocardiogr 2011;12:953-960.

43. Lewis AB, Crouse VL, Evans W, Takahashi M, Siegel SE. Recovery of left ventricular function following discontinuation of anthracycline chemotherapy in children. Pediatrics 1981;68:67-72.

44. Kocabaş A, Kardelen F, Ertug H, et al. Assessment of earlyonset chronic progressive anthracycline cardiotoxicity in children: different response patterns of right and left ventricles. Pediatr Cardiol 2014;35:82-88. 
45. Christiansen JR, Massey R, Dalen H, et al. Right ventricular function in long-term adult survivors of childhood lymphoma and acute lymphoblastic leukaemia. Eur Heart J Cardiovasc Imaging 2016;17:735-741.

46. Hu HM, Zhang XL, Zhang WL, Huang DS, Du ZD. Detection of Subclinical Anthracyclines' Cardiotoxicity in Children with Solid Tumor. Chin Med J 2018;131:14501456.

47. Koestenberger M, Nagel B, Ravekes W, et al. Reference values of tricuspid annular peak systolic velocity in healthy pediatric patients, calculation of $\mathrm{z}$ score, and comparison to tricuspid annular plane systolic excursion. Am J Cardiol 2012;109:116-121.

48. Ganame J, Claus P, Uyttebroeck A, et al. Myocardial dysfunction late after low-dose anthracycline treatment in asymptomatic pediatric patients. J Am Soc Echocardiogr 2007;20:1351-1358.

49. Radu LE, Ghiorghiu IA, Dorobantu DM, et al. Right ventricular systolic longitudinal function decreases early after low dose doxorubicin treatment in children and is linked to the decrease in left ventricle ejection fraction. Romanian Journal of Cardiology 2018;28:174-181.

50. Rosca MG, Hoppel CL. Mitochondrial dysfunction in heart failure. Heart Fail Rev 2013;18:607-622.
51. Nagueh SF, Smiseth OA, Appletion CP, et al. Recommendations for the Evaluation of Left Ventricular Diastolic Function by Echocardiography: An Update from the American Society of Echocardiography and the European Association of Cardiovascular Imaging. Eur Heart J Cardiovasc Imaging 2016;17:1321-1360.

52. Cairello F, Pessano S, Morsellino V, Haupt R, Derchi M. Cardiotoxicity in children. In: Lestuzzi C, Oliva S, Ferraù F (eds). Manual of cardio-oncology: Cardiovascular care in the cancer patient. Springer. 2017;13:215-242.

53. Stoddard MF, Seeger J, Liddell NE, Hadley TJ, Sullivan DM, Kupersmith J. Prolongation of isovolumetric relaxation time as assessed by Doppler echocardiography predicts doxorubicin-induced systolic dysfunction in humans. J Am Coll Cardiol 1992;20:62-69.

54. Tjeerdsma G, Meinardi MT, van Der Graaf WT, et al. Early detection of anthracycline induced cardiotoxicity in asymptomatic patients with normal left ventricular systolic function: autonomic versus echocardiographic variables. Heart 1999;81:419-423.

55. Venturelli F, Masetti R, Fabi M, et al. Tissue Doppler Imaging for anthracycline cardiotoxicity monitoring in pediatric patients with cancer. Cardio-Oncology 2018;4. doi:10.1186/s40959-018-0032-3. 